\title{
Evidence-Based PET for Cutaneous, Musculoskeletal and Unknown Primary Tumours
}

\author{
Luisa Knappe and Gaetano Paone
}

\subsection{Introduction}

PET/CT is extremely useful method in cutaneous and musculoskeletal tumours. In this chapter, the evidence from the literature concerning PET or $\mathrm{PET} / \mathrm{CT}$ in melanoma, sarcomas, bone metastases, cancer of unknown primary (CUP) and paraneoplastic syndromes were analysed.

\subsection{PET in Malignant Melanoma}

\subsubsection{Introduction}

Melanoma is a highly malignant tumour having its origin in melanocytes from the epidermal skin layer. As prognosis is highly dependent on lymph node involvement and presence of distant metastases at the time of diagnosis, a precise staging is important for determining prognosis and choosing the fittest therapy for the patient [1-4].

L. Knappe $\cdot$ G. Paone $(\bowtie)$

Clinic of Nuclear Medicine and Molecular Imaging, Imaging Institute of Southern Switzerland, Ente Ospedaliero Cantonale, Bellinzona and Lugano, Switzerland e-mail: gaetano.paone@eoc.ch

\subsubsection{Staging}

${ }^{18} \mathrm{~F}-\mathrm{FDG}$ PET/CT in staging melanoma has to be performed as a whole body protocol from head to toe to visualize the whole skin. Nonetheless, the usefulness of ${ }^{18} \mathrm{~F}$-FDG PET/CT is limited in staging of tumour expansion and detection of satellite metastases. For the recognition of lymph nodal metastases, ultrasound and histological examination of the sentinel lymph node have a higher sensitivity and specificity than ${ }^{18} \mathrm{~F}-\mathrm{FDG}$ PET/CT [5, 6]. Vural Topuz et al. showed that ${ }^{18} \mathrm{~F}-\mathrm{FDG}$ PET/ $\mathrm{CT}$ is probably negative in the first year postsurgery if the sentinel lymph node biopsy was negative. Hence, performance of ${ }^{18} \mathrm{~F}-\mathrm{FDG}$ PET/ $\mathrm{CT}$ is not recommended in early stage melanoma for not providing any significant clinical contribution [7].

In contrast, ${ }^{18} \mathrm{~F}-\mathrm{FDG}$ PET/CT is well established for imaging of distant metastases. In a meta-analysis of nine studies, Rodriguez Rivera et al. found out usefulness of ${ }^{18} \mathrm{~F}$-FDG PET/CT in staging of stage III melanoma having a high sensitivity $(89.4 \%)$ and specificity $(88.8 \%)$ [8]. Also Xing et al. valued ${ }^{18} \mathrm{~F}-\mathrm{FDG}$ PET/CT as the most sensitive and specific method for detecting distant metastases [6]. ${ }^{18} \mathrm{~F}-\mathrm{FDG} \mathrm{PET} / \mathrm{CT}$ is even superior to morphologic imaging and has replaced $\mathrm{CT}$ and magnetic resonance imaging (MRI) almost completely [1]. 


\subsubsection{Restaging and Treatment Monitoring}

The early detection of disease progression or recurrence has a huge impact on prognosis of melanoma. The usefulness of ${ }^{18} \mathrm{~F}$-FDG PET/CT has been proven not only for the staging of advanced melanoma but also for the detection of recurrences showing a sensitivity of $96 \%$ and a specificity of $92 \%$ [7]. Accordingly, ${ }^{18} \mathrm{~F}-\mathrm{FDG}$ PET/CT frequently leads to a change of treatment plan [6]. Due to a scarce number of prospective studies regarding use of ${ }^{18} \mathrm{~F}-\mathrm{FDG}$ PET/ $\mathrm{CT}$ in melanoma, more studies are needed to find the most effective and cost-effective intervals in follow-up.

\subsection{PET in Sarcomas}

\subsubsection{Introduction}

Sarcomas are malignant tumours originating from mesenchymal cells. They are a relatively rare cancer and represent only $1 \%$ of all malignant tumours but extremely frequent in children. They can be divided in soft tissue, osseous and chondral sarcomas. Soft tissue sarcomas are a group of heterogeneous tumours as rhabdomyosarcoma, leiomyosarcoma, fibrosarcoma, liposarcoma, angiosarcoma, etc. and they are the fourth most common solid tumours in children. The bone sarcomas are the osteosarcoma and the Ewing sarcoma. Classical imaging methods for sarcomas are X-ray, CT for control of stability and MRI for the illustration of the expansion in soft tissues. Biopsy and histopathological examination ensure diagnosis $[9,10]$.

\subsubsection{Staging}

PET/CT offers the possibility of simultaneous acquisition of bone lesions and their expansion in soft tissue and is very useful for the staging of sarcomas due to its high sensitivity, specificity and accuracy. The performance of ${ }^{18} \mathrm{~F}$-FDG PET/ $\mathrm{CT}$ in the initial staging provides information of the initial metabolism activity of the tumour. This is important for the follow-up and the evaluation of the therapy response [11]. About ${ }^{18} \mathrm{~F}-\mathrm{FDG}$ PET/CT in sarcomas, according to evidencebased data, the values vary between 86 and $96 \%$ for sensitivity and from 80 to $96 \%$ for specificity [12-15]. In particular, this hybrid imaging method is very useful for detecting distant metastases, as osseous and lung metastases [12, 15]. Furthermore, ${ }^{18} \mathrm{~F}-\mathrm{FDG}$ PET/CT might have a relevant impact on the development of treatment strategy plan [15]. Additional to the high diagnostic quality, ${ }^{18} \mathrm{~F}-\mathrm{FDG}$ PET/CT has also a prognostic value in sarcomas. Chen et al. found out that semi-quantitative PET parameters showed a significant prognostic value for overall survival and thus are useful tools in identifying high-risk patients [16]. These findings were confirmed by other authors reporting that a high maximum standardized uptake value (SUVmax) may predict a significantly shorter overall survival period [17].

\subsubsection{Restaging and Treatment Monitoring}

${ }^{18} \mathrm{~F}-\mathrm{FDG}$ PET/CT is a valuable method for detecting post-surgery recurrence in patients with sarcomas [12]. Liu et al. found a sensitivity of $92 \%$ and a specificity of $93 \%$ for the detection of recurrence in sarcoma [14]. Hongtao et al. reported that ${ }^{18} \mathrm{~F}$-FDG PET/CT is valuable for predicting the histological response to chemotherapy as they found a response to neoadjuvant chemotherapy in osteosarcomas with a sensitivity of $73 \%$ and a specificity of $86 \%$ [18]. Muheremu et al. showed that ${ }^{18} \mathrm{~F}-\mathrm{FDG}$ PET/CT assesses the efficacy of neoadjuvant therapy with a sensitivity and specificity of $79 \%$ and thus is a reliable imaging method not only in diagnosis but also in treatment control of osseous and soft tissue tumours [13]. Also Chen et al. valued posttreatment SUVmax as useful in monitoring therapy response [16]. Li et al. had similar results confirming that SUVmax before and after chemotherapy has effective prognostic significance for survival outcomes [19]. 


\subsection{PET for Bone Metastases}

\subsubsection{Introduction}

Bone metastases originate most frequently from breast cancer in women and from prostate carcinoma in men (each 60\%) followed by lung carcinoma (25\%), renal cell and thyroid carcinoma. Bone metastases can be differentiated in osteoblastic metastases which are typical for the prostate carcinoma, osteolytic metastases which occur in renal cell, thyroid or colon carcinomas and mixed osteoblastic and osteolytic metastases for example from breast or lung cancer. They are localized often in the spine (60\%) but also in the pelvis, proximal femur and skull, rarely in distal bones. Symptoms are mainly pain, radicular symptoms if a spine metastasis causes nerve root compression and functional impairment. Furthermore, metastases can cause instability of the bone with the consequent risk of fracture [20].

\subsubsection{Detection of Bone Metastases}

The probably most frequently performed imaging method for osseous staging is the bone scintigraphy (BS). In the actual development, this method is being replaced by PET/CT with different tracers as ${ }^{18} \mathrm{~F}-\mathrm{FDG}$ (which has the advantage to represent nearly all body districts) and ${ }^{18} \mathrm{~F}$-Fluoride which is more osseous specific. In a meta-analysis, Liu et al. found a sensitivity of $93 \%$ and a specificity of $95 \%$ for ${ }^{18} \mathrm{~F}$-fluoride $\mathrm{PET} / \mathrm{CT}$ in the detection of bone metastases. This method showed significantly higher sensitivity and specificity compared to BS and thus a superior diagnostic performance in bone metastases detection and higher accuracy [21]. Shen et al. achieved similar results showing a sensitivity of $92 \%$ and a specificity of $93 \%$ for ${ }^{18} \mathrm{~F}$-fluoride PET/CT. Compared with BS, it showed both higher sensitivity and specificity, whereas compared with ${ }^{18} \mathrm{~F}$-FDG PET/CT it showed only a higher sensitivity but no significant difference in specificity. Consequently, the authors describe an excellent diagnostic capacity for the detection of bone metastases and advantages compared with BS and ${ }^{18}$ F-FDG PET/CT [22].

Duo et al. analysed the performance of PET/ CT with different tracers in comparison with gadolinium-enhanced MRI for detecting bone metastases: similar sensitivity and specificity values for each method were found and consequently an excellent diagnostic performance for the detection of bone metastases for both methods [23]. On the contrary, regarding only vertebral metastases, MRI showed a better performance than PET/CT both in sensitivity and specificity. This procedure outranged also all other imaging methods as CT or BS with tomographic acquisition (SPECT) [24].

Concerning the prognostic value of ${ }^{18} \mathrm{~F}$-FDG PET/CT, Jeong et al. showed that patients with solid tumours and a lower level of ${ }^{18} \mathrm{~F}-\mathrm{FDG}$ uptake in the bone marrow have a better event free and overall survival than patients with higher bone marrow ${ }^{18} \mathrm{~F}$-FDG uptake and therefore propose to use the ${ }^{18} \mathrm{~F}$-FDG uptake in the bone marrow for risk stratification of tumour progression [25].

\subsection{PET for Cancer of Unknown Primary (CUP) and Paraneoplastic Syndromes}

\subsubsection{Introduction}

Cancer of unknown primary (CUP) is a syndrome defined by the presence of a metastatic disease without identified primary tumour. In $2-5 \%$ of all malignant tumours, the localization of the primary tumour is unknown. CUP occurs in a heterogeneous group of cancers most frequently in malignant melanoma, neuroendocrine tumours, carcinoids, small cell lung carcinoma and head and neck cancer. Despite of modern imaging methods, CUP remains a challenge in clinical routine. As prognosis is rather poor, the identification of the primary tumour can be important to adjust therapy and improve survival [26].

Paraneoplastic syndromes arise from tumour secretion of hormones, peptides or cytokines or 
from immune cross-reactivity between malignant and normal tissues. Paraneoplastic syndromes may affect diverse organ systems, most notably the endocrine, neurologic, dermatologic, rheumatologic and hematologic systems. The most commonly associated malignancies include small cell lung cancer, breast cancer, gynaecologic tumours and hematologic malignancies. In some instances, the timely diagnosis of these conditions may lead to detection of an otherwise clinically occult tumour at an early and highly treatable stage [27].

\subsubsection{Impact of PET in Patients with CUP}

Since patients with CUP syndrome usually underwent a vast diagnostic procedure with negative results, the patients setting in which ${ }^{18} \mathrm{~F}-\mathrm{FDG}$ $\mathrm{PET} / \mathrm{CT}$ is performed with this question is highly selected. Consequently, the search for a primary tumour in CUP syndrome is more difficult than in other diseases. Nevertheless, Burglin et al. found a detection rate of unknown primary tumours in $41 \%$ of cases by using ${ }^{18} \mathrm{~F}$-FDG PET/CT and they recommended an early use of ${ }^{18} \mathrm{~F}-\mathrm{FDG}$ PET/CT to obviate too much useless diagnostic procedures [28].

\subsubsection{Impact of PET in Patients with Paraneoplastic Syndromes}

In patients with suspected paraneoplastic syndrome, ${ }^{18}$ F-FDG PET/CT showed a high accuracy for the detection of underlying malignancies with a sensitivity of $81 \%$ and a specificity of $88 \%$ [29]. Also in patients with paraneoplastic neurological syndrome, ${ }^{18} \mathrm{~F}$ FDG PET/CT showed a high diagnostic performance with a detection rate of $15 \%$, a sensitivity of $87 \%$ and specificity of $86 \%$ [30]. Generally, a heterogeneity in study design and diagnostic workup and the small number of patients in the available studies reduce interpretability of the data $[29,30]$.

\section{References}

1. Perng P, Marcus C, Subramaniam RM. (18)F-FDG PET/CT and melanoma: staging, immune modulation and mutation-targeted therapy assessment, and prognosis. AJR Am J Roentgenol. 2015;205(2):259-70.

2. Deutsche Krebsgesellschaft (DKG), Deutsche Dermatologische Gesellschaft (DDG): S3-Leitlinie Diagnostik, Therapie und Nachsorge des Melanoms. 2016.

3. Larkin J, Chiarion-Sileni V, Gonzalez R, Grob JJ, Rutkowski P, Lao CD, et al. Five-year survival with combined nivolumab and ipilimumab in advanced melanoma. N Engl J Med. 2019;381:1535-46.

4. Danielsen M, Højgaard L, Kjær A, Fischer BM. Positron emission tomography in the followup of cutaneous malignant melanoma patients: a systematic review. Am J Nucl Med Mol Imaging. 2013;4(1):17-28.

5. Mirk P, Treglia G, Salsano M, Basile P, Giordano A, Bonomo L. Comparison between F-fluorodeoxyglucose positron emission tomography and sentinel lymph node biopsy for regional lymph nodal staging in patients with melanoma: a review of the literature. Radiol Res Pract. 2011;2011:912504.

6. Xing Y, Cromwell KD, Cormier JN. Review of diagnostic imaging modalities for the surveillance of melanoma patients. Dermatol Res Pract. 2012;2012:941921.

7. Vural Topuz Ö, Görtan FA, Kaya Döner ZR, Önsel Ç, Sayman HB. Usefulness of ${ }^{18}$ F-FDG PET/CT in cutaneous melanoma patients with negative sentinel lymph nodes and high Clark levels. Mol Imaging Radionucl Ther. 2018;27(2):66-72.

8. Rodriguez Rivera AM, Alabbas H, Ramjaun A, Meguerditchian AN. Value of positron emission tomography scan in stage III cutaneous melanoma: a systematic review and meta-analysis. Surg Oncol. 2014;23(1):11-6.

9. Doyle LA. Sarcoma classification: an update based on the 2013 World Health Organization classification of tumors of soft tissue and bone. Cancer. 2014;120(12):1763-74.

10. Amankwah EK, Conley AP, Reed DR. Epidemiology and therapies for metastatic sarcoma. Clin Epidemiol. 2013;5:147-62.

11. Katal S, Gholamrezanezhad A, Kessler M, Olyaei M, Jadvar H. PET in the diagnostic management of soft tissue sarcoma of musculoskeletal origin. PET Clin. 2018;13(4):609-2.

12. Huang T, Li F, Yan Z, Ma Y, Xiong F, Cai X, et al. Effectiveness of ${ }^{18} \mathrm{~F}-\mathrm{FDG}$ PET/CT in the diagnosis, staging and recurrence monitoring of Ewing sarcoma family of tumors: a meta-analysis of 23 studies. Medicine. 2018;97(48):e13457.

13. Muheremu A, Ma J, Amudong A, Ma Y, Niyazi M, $\mathrm{Ou} \mathrm{Y}$, et al. Positron emission tomography/computed tomography for osseous and soft tissue sarcomas: a systematic review of the literature and meta-analysis. Mol Clin Oncol. 2017;7(3):461-7. 
14. Liu F, Zhang Q, Zhu D, Li Z, Wang B, Zhou D, et al. Performance of positron emission tomography and positron emission tomography/computed tomography using fluorine-18-fluorodeoxyglucose for the diagnosis, staging, and recurrence assessment of bone sarcoma: a systematic review and meta-analysis. Medicine. 2015;94(36):e1462.

15. Treglia G, Salsano M, Stefanelli A, Mattoli MV, Giordano A, Bonomo L. Diagnostic accuracy of ${ }^{18} \mathrm{~F}-\mathrm{FDG}-\mathrm{PET}$ and $\mathrm{PET} / \mathrm{CT}$ in patients with Ewing sarcoma family tumours: a systematic review and a meta-analysis. Skelet Radiol. 2012;41(3):249-56.

16. Chen L, Wu X, Ma X, Guo L, Zhu C, Li Q. Prognostic value of 18F-FDG PET-CT-based functional parameters in patients with soft tissue sarcoma: a metaanalysis. Medicine. 2017;96(6):e5913.

17. Kubo T, Furuta T, Johan M, Ochi M. Prognostic significance of (18)F-FDG PET at diagnosis in patients with soft tissue sarcoma and bone sarcoma; systematic review and meta-analysis. Eur $\mathrm{J}$ Cancer. 2016;58:104-11.

18. Hongtao L, Hui Z, Bingshun W, Xiaojin W, Zhiyu W, Shuier Z, et al. 18F-FDG positron emission tomography for the assessment of histological response to neoadjuvant chemotherapy in osteosarcomas: a metaanalysis. Surg Oncol. 2012;21(4):e165-70.

19. Li YJ, Dai YL, Cheng YS, Zhang WB, Tu CQ. Positron emission tomography (18)F-fluorodeoxyglucose uptake and prognosis in patients with bone and soft tissue sarcoma: a meta-analysis. Eur J Surg Oncol. 2016;42(8):1103-14.

20. Grávalos C, Rodríguez C, Sabino A, Segui MA, Virizuela JA, Carmona A, et al. SEOM clinical guideline for bone metastases from solid tumours. Clin Transl Oncol. 2016;18(12):1243-53.

21. Liu Y, Sheng J, Dong Z, Xu Y, Huang Q, Pan D, et al. The diagnostic performance of 18F-fluoride PET/CT in bone metastases detection: a meta-analysis. Clin Radiol. 2019;74(3):196-206.
22. Shen CT, Qiu ZL, Han TT, Luo QY. Performance of 18F-fluoride PET or PET/CT for the detection of bone metastases: a meta-analysis. Clin Nucl Med. 2015;40(2):103-10.

23. Duo J, Han X, Zhang L, Wang G, Ma Y, Yang Y. Comparison of FDG PET/CT and gadoliniumenhanced MRI for the detection of bone metastases in patients with cancer: a meta-analysis. Clin Nucl Med. 2013;38(5):343-8.

24. Liu T, Wang S, Liu H, Meng B, Zhou F, He F, et al. Detection of vertebral metastases: a meta-analysis comparing MRI, CT, PET, BS and BS with SPECT. J Cancer Res Clin Oncol. 2017;143(3):457-65.

25. Jeong SY, Kim SJ, Pak K, Lee SW, Ahn BC, Lee J. Prognostic value of $18 \mathrm{~F}$-fluorodeoxyglucose bone marrow uptake in patients with solid tumors: a metaanalysis. Medicine. 2018;97(43):e12859.

26. Varadhachary GR. Carcinoma of unknown primary origin. Gastrointest Cancer Res. 2007;1(6):229-35.

27. Pelosof LC, Gerber DE. Paraneoplastic syndromes: an approach to diagnosis and treatment. Mayo Clin Proc. 2010;85(9):838-54.

28. Burglin SA, Hess S, Høilund-Carlsen PF, Gerke O. 18F-FDG PET/CT for detection of the primary tumor in adults with extracervical metastases from cancer of unknown primary: a systematic review and metaanalysis. Medicine. 2017;96(16):e6713.

29. Sheikhbahaei S, Marcus CV, Fragomeni RS, Rowe SP, Javadi MS, Solnes LB. Whole-body 18F-FDG PET and 18F-FDG PET/CT in patients with suspected paraneoplastic syndrome: a systematic review and meta-analysis of diagnostic accuracy. J Nucl Med. 2017;58(7):1031-6.

30. García Vicente AM, Delgado-Bolton RC, Amo-Salas M, López-Fidalgo J, Caresia Aróztegui AP, García Garzón JR, et al. 18F-fluorodeoxyglucose positron emission tomography in the diagnosis of malignancy in patients with paraneoplastic neurological syndrome: a systematic review and meta-analysis. Eur J Nucl Med Mol Imaging. 2017;44(9):1575-87.

Open Access This chapter is licensed under the terms of the Creative Commons Attribution 4.0 International License (http://creativecommons.org/licenses/by/4.0/), which permits use, sharing, adaptation, distribution and reproduction in any medium or format, as long as you give appropriate credit to the original author(s) and the source, provide a link to the Creative Commons license and indicate if changes were made.

The images or other third party material in this chapter are included in the chapter's Creative Commons license, unless indicated otherwise in a credit line to the material. If material is not included in the chapter's Creative Commons license and your intended use is not permitted by statutory regulation or exceeds the permitted use, you will need to obtain permission directly from the copyright holder.

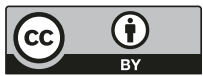

